\title{
REPRODUCTIVE BIOLOGY
}

\section{Sperm size evolution}

Sperm length unexpectedly varies more than 3,000-fold across species, posing new questions for anisogamy theory and understanding the different forces shaping evolution of the male gamete.

\section{Matthew J. G. Gage}

School of Biological Sciences, University of East Anglia, Norwich Research Park, NORWICH NR4 7TJ United Kingdom

m.gage@uea.ac.uk

Sperm have one primary job: to carry a male's DNA to unite with a conspecific egg, form a zygote, and enable sexual reproduction. Given this uniform task, why are spermatozoa among the most structurally diverse cell types ${ }^{1}$ ? Writing in Nature Ecology and Evolution, Kahrl et al. ${ }^{2}$ demonstrate an astounding size variation among sperm, while providing some fundamental answers to why sperm show such wide variation andbuilding an important foundation for future research into the evolution of sperm form and function.

Before examining what forces shape such profound variation in the male gamete, it is worth considering how sperm and egg came to be. Males make sperm, and females make eggs, and this binary strategy for achieving sexual reproduction probably evolved more than a billion years ago, evidenced by fossil remains containing both gamete types in the ancient algae Bangiomorpha pubsecens $^{3}$. The evolution of anisogamy (different gamete types) is theorised to have evolved via an unusual force known as disruptive selection ${ }^{4}$, where two equally optimal strategies to achieve one goal exist within a species. As today, life's first protogametes had two fundamental roles as they dispersed into Earth's primordial waters to achieve sexual reproduction: to win fusions with protogametes of the same species, and to carry sufficient resources to nourish the embryo into a viable hatchling ${ }^{4,5}$. The problem is that these two tasks demand opposing gamete phenotypes, in which size and number trade against each other (Figure 1). To resource the embryo, gametes need to be large, which will limit number production. To win fusions against competition or dilution, 'astronomical' numbers should be released into the fertilisation lottery, limiting gamete size 5 . Disruptive selection about these two divergent optima for either nourishing the embryo or winning fertilisations can therefore explain the anisogamous evolution of a few large eggs, or many tiny sperm, and consequently females and males ${ }^{4,5}$. 
Anisogamy theory therefore raises an important question for sperm evolution: if numerical competition for fertilisations drives up number at the expense of size ${ }^{4,5}$, should sperm not be typically of minimal size across all taxa? The answer, conclusively provided by Kahrl et al. ${ }^{2}$ is no. The authors show a counterintuitively enormous 3,000-fold variation in sperm length across species, ranging from microcells to gametic giants. Following deep searching across a wide scientific literature, $\underline{K a h r l}$ et al. ${ }^{2}$ collated the world's biggest and most complete sperm length dataset, reporting on 3,233 species across 21 animal phyla, from simple sponges to complex chordates. Using this impressive dataset, they reveal huge sperm size variation, from minuscule 2 micrometer male sex cells of Branchionus bidentatus rotifers, to gametic goliaths in Drosophila bifurca fruit flies that are almost $6 \mathrm{~cm}$ long, far greater than their male producers.

The authors use this extensive dataset to answer some fundamental questions about what explains variation in sperm length. Mapping the species onto the Open Tree of Life phylogeny ${ }^{6}$, they performed careful comparative analyses to explore how the many transitions from external to internal fertilisation modes have influenced the direction and rate of sperm length evolution. Across 3,166 species and multiple evolutionary paths between fertilisation modes, the study revealed that sperm become much bigger following the transition to internal fertilisation, growing over six-fold to an average 420 micrometers, compared to 61 micrometers across external fertilisers. Calibration of the phylogeny also showed that internal fertilisation was associated with a three-fold increase in sperm diversification rate compared to external fertilisation, and accounting for $90 \%$ of the major adaptive shifts in sperm length².

Kahrl et al. therefore document extreme divergence in sperm size across the animal kingdom, and reveal that sperm get bigger and evolve faster when fertilisation mode changes from external to internal. The authors suggest a number of plausible hypotheses for these major changes, and their study provides an important basis for further testing. Although fertilisation competitions may still follow a numbers game, a more complex and heterogenous internal sperm operating environment may have added additional or stronger selective pressures on spermatozoal phenotypes. External fertilisation is likely to encourage sperm dilution compared with the more tightly contained environment of the female tract, perhaps selecting more on sperm number for winning scramble competitions in a more dispersed gamete fusion lottery. By contrast, internal fertilisation may apply tighter spatial selection on sperm form and function within a confined female tract, encouraging evolution of sperm 'quality' as well as 'quantity', perhaps reflected by sperm elongation and 
diversification. Consistent with these ideas, the study found that there were more adaptive shifts in sperm length for internally-fertilising arthropods with small reproductive tract volumes, compared to those inside larger-bodied vertebrates.

Just as sexual selection shapes reproductive evolution in adult males and females, it also contributes to post-mating competition and choice at the gamete level ${ }^{7}$. Most species exhibit multiple mating ${ }^{8}$, so sperm from different males will mix and compete for fertilisation, while giving females opportunities to evolve complex competitive arenas and mechanisms of cryptic choice for the 'right' sperm $^{9}$. It is clear from remarkable in vivo videos of transgenic fluorescent sperm within Drosophila female tracts that there is a lot going on at this critical gamete level, and stored sperm are not sitting passively, waiting their turn for fusion ${ }^{10}$. It is also clear that female tracts are not simple vessels for encouraging sperm to the egg, but that remarkable complexity has evolved ${ }^{11}$, consistent with mechanisms of female sperm selection for good or compatible paternal genes ${ }^{9}$. Sexual selection has caused the evolution of weird and wonderful traits in adult males and females, so similar forces operating after mating will encourage the evolution of gametic equivalents of exaggerated plumage, antlers, horns or teeth ${ }^{12}$, and complex arenas for competition. Kahrl et al. provide a robust framework and huge dataset for biologists to go forth and chase up exciting questions about sperm length evolution in new taxa. How does sperm form relate to function? How do different fertilisation environments shape sperm phenotypes, such as fresh- versus salt-water, or hot- versus coldblooded reproductive tracts? How does sperm morphology co-vary with body size, or genome size? How is sperm length genetically controlled? And how do different mechanisms of sperm competition and cryptic female choice shape sperm evolution? Finally, although this study provides us with the world's biggest dataset, 3,233 species is a tiny proportion of Earth's multi-million animal species, presenting opportunities for researchers to go forth right now to discover even more extreme and informative spermatozoal diversity.

\section{REFERENCES}

1. Pitnick, S., Hosken, D. J. \& Birkhead, T. R. Sperm morphological diversity. pp. 69-149 in T. R. Birkhead, D. J. Hosken, and S. S. Pitnick, eds. Sperm biology: an evolutionary perspective. Academic Press, London (2009).

2. Kahrl, A.F., Snook, R.R. \& Fitzpatrick J.L. Fertilization mode drives sperm length evolution across the animal tree of life. DOI TBC Nat. Ecol. \& Evol. (2021).

3. Butterfield, N.J. Bangiomorpha pubescens: implications for the evolution of sex, multicellularity, and the Mesoproterozoic/Neoproterozoic radiation of eukaryotes. Paleobiol. 26, 386-404 (2000). 
4. Parker, G. A., Baker, R. R. \& Smith V.L. The origin and evolution of gamete dimorphism and the malefemale phenomenon. J. Theor. Biol. 36, 529-553 (1972).

5. Parker, G. A. Why are there so many tiny sperm? Sperm competition and the maintenance of two sexes. J. Theor. Biol. 96, 281-294 (1982).

6. Hinchliff, C. E. et al. Synthesis of phylogeny and taxonomy into a comprehensive tree of life. Proc. Natl. Acad. Sci. U.S.A. 112, 12764-12769 (2015).

7. Parker, G.A. Sperm competition and its evolutionary consequences in the insects. Biol. Rev. 45, 525567 (1970).

8. Taylor, M. L., Price, T. A. R. \& Wedell, N. Polyandry in nature: a global analysis. Trends Ecol. Evol. 29, 376-383 (2014).

9. Eberhard, W. Female control: sexual selection by cryptic female choice. (Princeton University Press, 1996).

10. Manier, M.K., Belote, J.M., Berben, K.S., Novikov, D., Stuart, W.T. \& Pitnick, S. Resolving mechanisms of competitive fertilization success in Drosophila melanogaster. Science 328, 354-357 (2010).

11. Scott Pitnick, S., Wolfner, M.F \& Suarez, S.S. Ejaculate-female and sperm-female interactions. pp. 247-304 in T. R. Birkhead, D. J. Hosken, and S. S. Pitnick, eds. Sperm biology: an evolutionary perspective. Academic Press, London (2009).

12. Lupold, S., Manier, M. K., Puniamoorthy, N., Schoff, C., Starmer, W.T., Luepold, S.H.B., Belote, J.M. \& Pitnick, S. How sexual selection can drive the evolution of costly sperm ornamentation. Nature 533, 535538 (2016).

\section{Figure legend}

\section{Figure 1. Evolution of anisogamy.}

Disruptive selection for either numerous competitive gametes or large nutritive gametes can explain the evolution of anisogamy, or sperm and egg (and male and female). The evolutionary stability of these two strategies can be maintained by numerical sperm competition: a doubling in sperm size to nourish the zygote remains an inconsequential contribution to offspring fitness, but a consequential halving of sperm numbers for winning fertilisation competitions. Adapted from Parker et al (1972).

Competing interests: The author declares no competing interests. 